\title{
Doripenem in hospital infections: a focus on nosocomial pneumonia, complicated intra-abdominal infections, and complicated urinary tract infections
}

This article was published in the following Dove Press journal:

Infection and Drug Resistance

29 June 2009

Number of times this article has been viewed

\author{
Tze Shien Lo' \\ Stephanie M Borchardt ${ }^{2}$ \\ Justin $\mathrm{MWelch}^{3}$ \\ Melissa A Rohrich ${ }^{3}$ \\ Augusto M Alonto ${ }^{4}$ \\ Anne $\mathrm{V}$ Alonto ${ }^{5}$ \\ 'Infectious Diseases Service, Veterans \\ Administration Medical Center, \\ Fargo, North Dakota, USA; ${ }^{2}$ Research \\ Service, Veterans Administration \\ Medical Center, Fargo, North Dakota, \\ USA; ${ }^{3}$ Pharmacy Service, Veterans \\ Administration Medical Center, Fargo, \\ North Dakota, USA; ${ }^{4}$ Infectious \\ Diseases Department, MeritCare \\ Medical Center, Fargo, North Dakota, \\ USA; ${ }^{5}$ Department of Internal \\ Medicine, University of North Dakota \\ School of Medicine and Health \\ Sciences, Fargo, North Dakota, USA
}

Correspondence:Tze Shien Lo VA Medical Center (IIC-I), 210 I Elm Street, Fargo ND 58102, USA $\mathrm{Tel}+\mathrm{I}$ 70I-239-3700 Ext. 9-3509 Fax +I (70I) 237-249I Email tshienlo@medicine.nodak.edu

\begin{abstract}
Doripenem is the latest carbapenem on the market to date. Although not an antibiotic in a new class, it offers a glimmer of hope in combating serious infections secondary to multidrug-resistant Gram-negative bacteria when we have not seen a new class of antibacterial, particularly for Gram-negative bacteria, for more than 10 years. In vitro, doripenem exhibits a broad spectrum of activity against Gram-positive and Gram-negative bacteria, including extended-spectrum $\beta$-lactamase (ESBL) and Amp-C $\beta$-lactamase producing Enterobacteriaceae and anaerobes. Doripenem also exhibits better in vitro activity against Pseudomonas aeruginosa compared to other anti-pseudomonal carbapenems. It combines the desirable activities of both imipenem and meropenem. It has similar activity to imipenem against Gram-positive pathogens and has the antimicrobial spectrum of meropenem against Gram-negative organisms. Several randomized clinical trials have demonstrated that doripenem is non-inferior to meropenem, imipenem, piperacillin/tazobactam, or levofloxacin in its efficacy and safety profile in treating a wide range of serious bacterial infections including intra-abdominal infection, complicated urinary tract infection, and nosocomial pneumonia. Due to its wide spectrum of activity and good safety profile it is susceptible to misuse leading to increasing rates of resistance. Judicious use should be considered when using doripenem as a first-line agent or drug of choice for serious infections. Doripenem is a well-tolerated drug with common adverse effects including headache, nausea and diarrhea. Caution should be used in patients with hypersensitivity to carbapenems and adverse reactions to $\beta$-lactam agents. Dosage adjustment is needed for patients with renal impairment. Doripenem has demonstrated economic and clinical benefits. It has been shown to reduce hospital length of stay and duration of mechanical ventilation for intensive care unit (ICU) patients. Therefore, doripenem is a welcome addition to our limited armamentarium of antibiotics available to treat serious bacterial infections in hospitalized patients.
\end{abstract}

Keywords: doripenem, nosocomial pneumonia, intra-abdominal infections, urinary tract infections

\section{Introduction}

The emergence of antimicrobial resistance among Gram-negative organisms, especially extended-spectrum $\beta$-lactamase (ESBL)-producing Enterobacteriaceae, and resistant Pseudomonas and Acinetobacter species, has been a significant challenge for the clinician. ${ }^{1} \beta$-lactams are one of the most widely prescribed antimicrobial agents in the hospital setting. However, their use has resulted in a dramatic increase in the selection of $\beta$-lactamase variants, which threatens the utility of this class of antimicrobial. ${ }^{2}$ The development and approval of carbapenems was a milestone in addressing this situation 
because of their broad-spectrum activity against Gram-positive, Gram-negative, and anaerobic bacteria. ${ }^{3}$

Multi-drug resistant Gram-negative bacteria have been a significant cause of morbidity and mortality especially for hospitalized patients. There is an ongoing need for effective pharmacotherapy against these microorganisms. Among these are Pseudomonas aeruginosa, Acinetobacter spp., specifically Acinetobacter baumannii and Klebsiella pneumoniae. Colistin is an agent which has been used as empiric treatment for metallo- $\beta$-lactamase-producing K. pneumoniae. There are three other agents, that are either in development or on the market, worth mentioning that possess antimicrobial activity against multi-drug resistant Gram-negative bacteria: RO49084631/CS-023, tigecycline and CP3242. ${ }^{4}$

RO49084631/CS023 is a carbapenem that has demonstrated activity against Gram-positive and Gram-negative aerobes and anaerobes such as methicillin-resistant Staphylococcus aureus (MRSA), methicillin-resistant Staphylococcus epidermidis (MRSE), penicillin-resistant Streptococcus pneumoniae (PRSP), $\beta$-lactamase-negative ampicillinresistant Haemophilus influenzae and $P$. aeruginosa. Tigecycline is a bacteriostatic agent which inhibits protein translation. It possesses activity against MSSA, MRSA and MRSE, as well as vancomycin-resistant Enterococcus spp. Lastly, CP3242 is an inhibitor of metallo- $\beta$-lactamases. It demonstrated potency against MBL-producing $P$. aeruginosa in vivo and in vitro when combined with biapenem, imipenem, meropenem or ceftazidime. ${ }^{4}$

Because the carbapenems have the broadest antibacterial spectrum of activity, there is concern that abuse or overuse of this class of antibiotic will lead to the development of resistance. ${ }^{5}$ Various methods have been suggested in an effort to either delay resistance selection or to enhance activity against resistant strains including the co-administration of an aminoglycoside with doripenem ${ }^{1}$ and prolonging antibiotic infusion, ${ }^{6}$ respectively.

Rising rates of antibiotic-resistance among bacteria in the hospital setting highlight the need for new therapeutic options, ${ }^{7}$ in particular, for the treatment of MRSA and other organisms which are resistant to doripenem. Although doripenem offers a glimmer of hope in combating serious hospital infections, the fact remains that only ten new antibiotics have been approved within the past ten years, of which only two have been truly novel. This highlights the growing concern over the present drought in the antibiotic research and development pipeline and, therefore, the need for further emphasis on drug discovery.

\section{Chemistry}

Doripenem is the newest member of the carbapenems on the US market. Human kidneys produce dehydropeptidase-1 (DHP-1), which can hydrolyze and inactivate imipenem. However, the addition of a methyl chain on the nucleus of doripenem increases its stability against DHP-1. ${ }^{8}$ This methyl side chain is also found in meropenem and ertapenem. Doripenem has a sulfamoly-aminomethyl-pyrrolidinylthio side chain replacing the dimethyl-carbamoyl-pyrrolidinylthio side chain in position 2 of meropenem. This unique side chain of doripenem enhances its potency against Gram-positive bacteria, such as methicillin-sensitive $S$. aureus, while maintaining its activity against Gram-negative bacteria. ${ }^{4}$

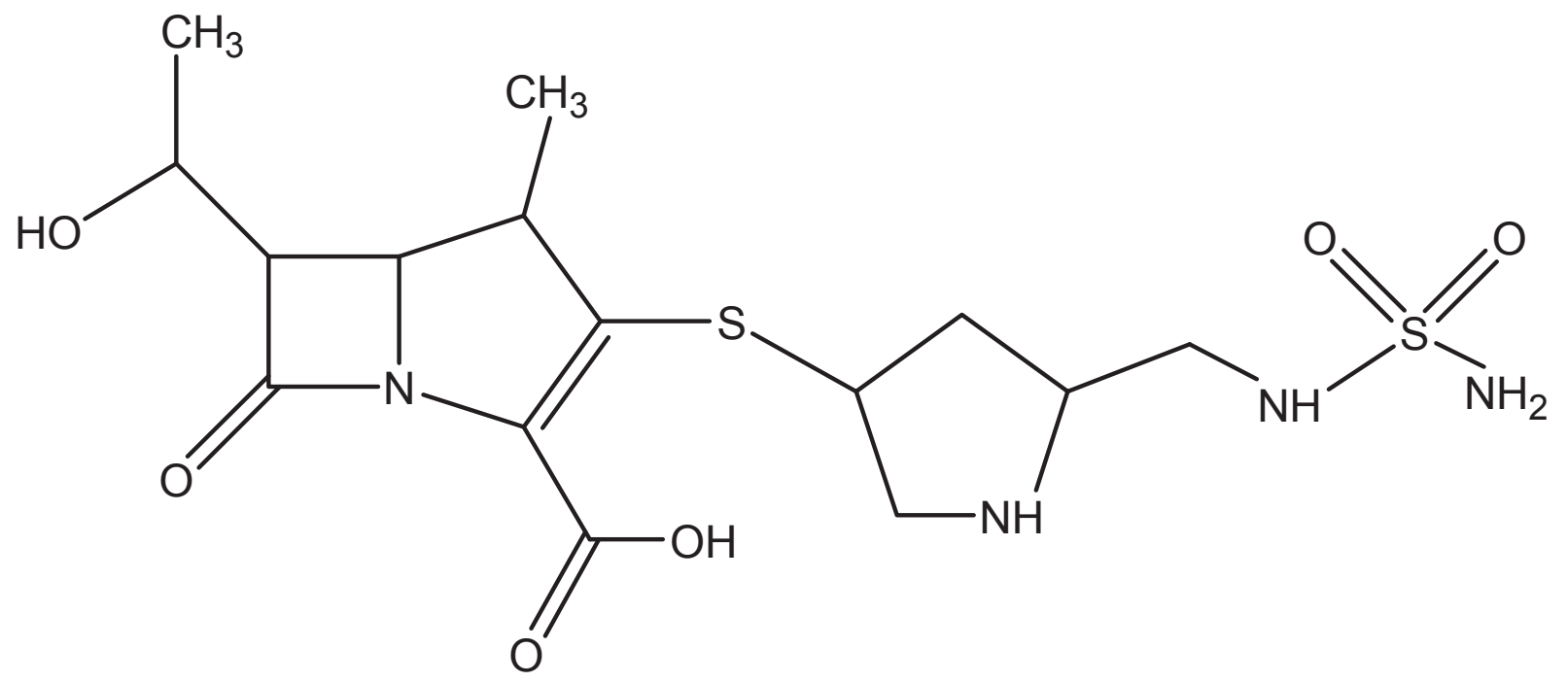

Figure I Chemical structure of doripenem. 


\section{Mode of action}

Like other $\beta$-lactams and carbapenems, doripenem exhibits its antimicrobial effects by binding penicillin binding proteins (PBP) that are necessary to maintain the bacterial cell wall leading to cell death. ${ }^{9}{ }^{10}$ Doripenem binds to PBP-2, PBP-3, and PBP-4. ${ }^{10}$ Affinities for PBPs vary by species. ${ }^{5}$

\section{Pharmacodynamics}

The bactericidal effects are nonconcentration dependent; the longer the time above the minimum inhibitory concentration (T > MIC), the greater the effect. ${ }^{5,11-14}$ The proportion of time during the dosing interval, where the concentration is greater than the MIC for non-protein bound carbapenems, correlates to in vivo activity of the drug for a specific organism. ${ }^{15} \mathrm{In}$ general, for carbapenems, a T > MIC of $20 \%$ results in a bacteriostatic effect with the maximum bactericidal effect at $40 \%{ }^{4,16}$ Katsube et al studied the $\% \mathrm{~T}>$ MIC for doripenem against three strains of $P$. aeruginosa. They showed a static effect with a $\mathrm{T}>\mathrm{MIC}$ at $25 \%, 23.9 \%$ and $39.8 \%$ and a 2-log killing effect at $28.1 \%, 29.5 \%$ and $49.6 \%$ and a $90 \%$ maximum killing effect at $36.5 \%, 46.8 \%$ and $80.7 \%$ for respective strains. ${ }^{17}$

\section{Spectrum of activity}

Doripenem has a broad spectrum of activity against Gram-positive, Gram-negative and anaerobic bacteria including multidrug-resistant strains, most notably the ESBL and Amp-C $\beta$-lactamase producing Gram-negative organisms. ${ }^{2,5,9-11,13,14,18}$ It has comparable antimicrobial activity to meropenem and imipenem. ${ }^{2,13,14}$ In vitro activity against $P$. aeruginosa, Acinetobacter spp., ceftazidimeresistant bacteria and selected ESBL-producing bacteria show lower MICs with doripenem. For example, Fritsche et al through analysis of the antimicrobial activity of 16,008 clinical isolates, demonstrated that doripenem was the most active agent $\left(\mathrm{MIC}_{90} 8 \mathrm{mg} / \mathrm{L}\right)$ among the carbapenems tested against wild type $P$. aeruginosa. In this study, the $\mathrm{MIC}_{90}$ of meropenem and ertapenem were found to be $16 \mathrm{mg} / \mathrm{L}$ and $>8 \mathrm{mg} / \mathrm{L}$, respectively. However, the clinical relevance is unknown. ${ }^{2,19}$ Doripenem has in vitro activity against $H$. influenzae (including $\beta$-lactamase positive isolates) and Moraxella catarrhalis. ${ }^{2,18}$

As with other carbapenems, doripenem is not active against Enterococcus faecium or MRSA because of alteration of the bacteria's PBPs leading to poor binding affinity. ${ }^{13,20}$

Although doripenem is stable against most $\beta$-lactamases, some carbapenemases will affect its activity. The Ambler's class B metallo- $\beta$-lactamase is a potent cabapenemase
Table I In vitro susceptibility of common pathogens to doripenem ${ }^{2,4,10}$

\section{Gram-positive microorganisms}

Streptococcus constellatus

Streptococcus intermedius

Streptococcus agalactiae Streptococcus pneumoniae

Streptococcus pyogenes

Methicillin-susceptible Staphylococcus aureus

Gram-negative microorganisms

Acinetobacter baumannii

Citrobacter freundii

Enterobacter aerogenes

Enterobacter cloacae

Escherichia coli (including ESBL confirmed spp.)

Haemophilus influenzae (including $\beta$-lactamase producing strains)

Klebsiella pneumoniae (including ESBL confirmed spp.)

Klebsiella oxytoca

Moraxella catarrhalis

Morganella morganii

Proteus mirabilis

Pseudomonas aeruginosa

Serratia marcescens

Anaerobic microorganisms

Bacteroides caccae

Bacteroides fragilis

Bacteroides thetaiotaomicron

Bacteroides uniformis

Bacteroides vulgatus

Peptostreptococcus micros

Abbreviation: ESBL, extended spectrum $\beta$-lactamase.

which is intrinsically produced by Stenotrophomonas maltophilia, resulting in resistance to carbapenems, including doripenem. However, some strains of Gram-negative bacteria, such as $P$. aeruginosa, Acinetobacter baumannii and Enterobacteriaceae, are resistant to doripenem by producing Ambler's class A enzymes, class B metallo- $\beta$-lactamases (IMP, VIM, SPM), class D enzymes (OXA group) and KPC enzymes. ${ }^{2,15,18,21,22}$

Recently, the incidence of carbapenemase-producing Enterobacteriaceae (CRE) including Klebsiella spp. has been increasing globally. Serine $\beta$-lactamase KPC is the main carbapenemase produced by CRE. Because CRE are highly resistant to doripenem (MIC 8 to $>64 \mu \mathrm{g} / \mathrm{ml}$ ) and other carbapenems, there is no reliable antibiotic treatment for patients infected with CRE, thus, causing high morbidity and mortality in hospitalized patients. ${ }^{23,24}$

P. aeruginosa and other Gram-negative bacteria can confer resistance to doripenem by overexpressing multidrug 
efflux pump system called MexA-MexB-OprM, resulting in decreased antibiotic concentration at target sites. ${ }^{25}$ These bacteria can also exhibit resistance to many antibiotics through a combination of increased permeability, efflux system and production of $\beta$-lactamases. One way of increasing permeability is through loss of OprD porin of the organisms. The sensitivity of $P$. aeruginosa to imipenem is reduced by loss of OprD porin; however, Mushtaq and co-workers demonstrated that a combination of loss of OprD porin and presence of efflux pump is necessary for $P$. aeruginosa to develop resistance against meropenem and doripenem. ${ }^{26}$

Susceptibility to doripenem was defined as $\leq 0.5 \mu \mathrm{g} / \mathrm{mL}$ for Enterobacteriaceae, $\leq 2 \mu \mathrm{g} / \mathrm{mL}$ for $P$. aeruginosa,$\leq 1 \mu \mathrm{g} / \mathrm{mL}$ for Acinetobacter baumannii and anaerobes, and $\leq 0.12 \mu \mathrm{g} / \mathrm{mL}$ for Streptococcus anginosus group. However, when susceptibility reports of doripenem are not available, Jones and colleagues reported carbapenems (imipenem, meropenem, ertapenem), oxacillin and ampicillin can be used as surrogate testing agents with high absolute categorical agreement $(89.1 \%$ to $100 \%)$ and negligible false-susceptible error $(<0.1 \%) .{ }^{27}$

\section{Pharmacokinetics}

Among a group of healthy study participants given a single 1-hour intravenous infusion of $500 \mathrm{mg}$, the mean plasma concentration $\max \left(\mathrm{C}_{\max }\right)$ was $23 \mu \mathrm{g} / \mathrm{mL}$ and the mean area under the curve (AUC) was $36.3 \mu \mathrm{g} \cdot \mathrm{h} / \mathrm{mL}$.

Doripenem has linear kinetics when infused intravenously over one hour over a dose range of $500 \mathrm{mg}$ to $1 \mathrm{~g} .{ }^{10}$ It has an $8.1 \%$ average binding to plasma proteins with a median volume of $16.8 \mathrm{~L}$ (8.09 tp $55.5 \mathrm{~L}$ ) among healthy participants. ${ }^{10}$ Doripenem also demonstrates significant penetration into several body fluids and tissues, including peritoneal and retroperitoneal fluids, urine, bile and the gallbladder. ${ }^{10}$

Doripenem is metabolized by dehydropeptidase-I to an inactive metabolite (doripenem-M1). It does not interact with the cytochrome P450 (CYP450) enzymes as either a substrate or an inhibitor. ${ }^{10}$ In healthy volunteers, $15 \%$ of the dose was recovered in urine as metabolite within 48 hours. ${ }^{10}$

The approved dosing and administration of doripenem is $500 \mathrm{mg}$ every 8 hours infused over one hour for patients $>18$ years of age for complicated intra-abdominal infections (cIAIs) or complicated urinary tract infections (cUTIs). ${ }^{10}$ Doripenem is eliminated primarily by the kidneys via glomerular filtration and active tubular secretion with $70 \%$ recovered in the urine as unchanged drug. ${ }^{10}$ The halflife of doripenem is approximately 1 hour among healthy adults. ${ }^{10}$ Doripenem dose adjustment for renal impairment is required.
Dosage adjustments are not recommended for the elderly nor should they be based on gender. ${ }^{10}$ Doripenem is hemodialyzable with systemic levels reduced by $48 \%$ to $62 \%$. However, the manufacturer has not given recommendations for dose adjustments in hemodialysis due to insufficient information. ${ }^{10}$

Doripenem is stable in $0.9 \%$ sodium chloride for eight hours at room temperature and may exceed twelve hours. ${ }^{4,10,14}$

\section{Efficacy and safety of doripenem}

Doripenem was approved by the United States Food and Drug Administration (FDA) in October 2007 for the treatment of cIAIs and cUTIs, including pyelonephritis. ${ }^{4}$ The manufacturer (Johnson \& Johnson Pharmaceutical Research and Development) of doripenem (Doribax ${ }^{\circledR}$ ) has submitted an application for the additional indication of nosocomial pneumonia (NP), including ventilator-associated pneumonia, which is currently under FDA review. ${ }^{28}$ Doripenem is indicated for NP in the European Union. ${ }^{11}$

The following sections will discuss the clinical efficacy and safety of doripenem in treatment of cIAIs, cUTIs and NP.

\section{Complicated intra-abdominal infections (clAl)}

Two Phase 3 trials were conducted to establish the efficacy and safety of doripenem in treating intra-abdominal infections.

Lucasti et $\mathrm{al}^{29}$ conducted a prospective, multicenter, double blind trial which compared intravenous (IV) doripenem $500 \mathrm{mg}$ every 8 hours $(\mathrm{n}=237)$ with IV meropenem $1000 \mathrm{mg}$ every 8 hours $(n=239)$. Patients in both groups could be switched to oral amoxicillin/clavulanate after adequate clinical improvement was achieved. The primary endpoint was to compare the clinical cure rates of doripenem versus meropenem in treatment of cIAIs in hospitalized patients at the test of cure (TOC) visit. The TOC visit could range from 21 to 60 days after completing the study drug therapy. The second

Table 2 Pharmacokinetic properties of doripenem following a single I-hour intravenous infusion of a $500 \mathrm{mg}$ dose administered to healthy participants $(\mathrm{N}=24)^{10}$

\begin{tabular}{ll}
\hline Property & Value for doripenem \\
\hline Mean $\mathrm{C}_{\max }$ & $23 \mu \mathrm{g} / \mathrm{mL}$ \\
Mean AUC & $36.3 \mu \mathrm{g} \cdot \mathrm{h} / \mathrm{mL}$ \\
Protein binding & $8.1 \%$ \\
Volume of distribution & $16.8 \mathrm{~L}$ \\
Metabolism & Dehydropeptidase-I \\
Half-life & $\sim 1$ hour \\
\hline
\end{tabular}

Abbreviations: $C_{\max }$, maximum concentration; $A U C$, area under the curve. 
endpoints were to compare (1) clinical responses at the end of IV treatment, at an early follow-up visit; (2) microbiological responses at the end of IV treatment, early follow-up and TOC visits; and (3) safety profiles.

Comparison of clinical cure rates at the TOC visit showed doripenem was non-inferior to meropenem. Microbiological eradication (ME) rates for common pathogens (eg, Escherichia coli, P. aeruginosa) were not statistically different between the treatment groups.

The most common treatment related adverse reactions were nausea, fever, diarrhea, anemia and phlebitis for both carbapenems. There was no significant difference with regard to their safety profile.

Another prospective, multicenter, double blind trial conducted by Malafaia and colleagues ${ }^{30}$ was similar in design to the study by Lucasti et al. The researchers compared IV doripenem $500 \mathrm{mg}$ every 8 hours a day versus meropenem $1000 \mathrm{mg}$ every 8 hours a day in treating intra-abdominal infections. Patients in both groups were allowed to switch to oral amoxicillin/clavulanate after completing a minimum of 9 doses of IV antibiotics.

Four hundred and eighty-six patients were randomized to either the doripenem group or the meropenem group. The patients were first assessed for early efficacy and safety 7 to 14 days after the end of therapy. They were then assessed for microbiologic and clinical efficacy (CE) 28 to 42 days after the TOC visit. The primary endpoint was to compare clinical cure rates in the ME group at the TOC visit and in the microbiological modified intent-to-treat (mMITT) group. Secondary endpoint was to compare clinical cure rates in the $\mathrm{CE}$ group and microbiologic response in the ME group at the TOC visit. Doripenem was shown to be non-inferior to meropenem in achieving both primary and secondary endpoints.

Safety profile for both carbapenems was very similar and there were no deaths during the study that were attributable to any of the antibiotics. The most common adverse reactions related to doripenem were nausea, vomiting, diarrhea and anemia.

In essence, the outcomes of Malafaia's study were similar to Lucasti's with respect to efficacy and adverse drug events.

\section{Complicated urinary tract infections}

A Phase 3, prospective, multicenter, randomized double-blind trial conducted by Naber et $\mathrm{al}^{31}$ compared IV doripenem $500 \mathrm{mg}$ every 8 hours versus IV levofloxacin $250 \mathrm{mg}$ every 24 hours in the treatment of cUTI. Both antibiotics were administered as a 1-hour IV infusion. Seven hundred and fifty-three patients were enrolled, 377 in the doripenem arm and 376 in the levofloxacin arm. The primary endpoint is to determine the microbiologic response at the TOC visit, which is defined as 6 to 9 days after the completion of study drug therapy following a 10-day treatment regimen. The secondary objective is to determine the clinical response at the TOC visit. After $\geq 9$ doses of IV study drug therapy, patients could be switched to oral levofloxacin if the patient became afebrile, and had no signs or symptoms of cUTI and negative urine culture.

Analysis of the efficacy results revealed that doripenem was non-inferior to levofloxacin in microbiological and clinical effectiveness. Doripenem was effective against major causative organisms of cUTIs including; E. coli, Proteus mirabilis, and K. pneumoniae. Analysis of safety data indicated doripenem was well tolerated by patients without demonstrating serious adverse reactions, such as seizures. The most common side effect for this study was headache.

Another randomized, controlled, double-blind trial $(\mathrm{n}=155)$ was conducted in Japan by Kamidono et al ${ }^{32}$ to compare the efficacy of IV doripenem $250 \mathrm{mg}$ every 12 hours $(n=76)$ versus IV meropenem $500 \mathrm{mg}$ every 12 hours $(n=79)$ in patients with cUTIs requiring parenteral antibiotic therapy. All subjects were adult inpatients aged 20 to 79 years of age who demonstrated both pyuria and bacteriuria. Clinical efficacy was $93.4 \%$ (71/76) in the doripenem group versus $92.4 \%$ $(73 / 79)$ in the meropenem group. The bacteriologic response was $95.9 \%$ (94/98) in the doripenem group versus $96 \%$ $(101 / 105)$ in the meropenem group. The authors concluded that doripenem was non-inferior to meropenem, clinically and bacteriologically. In regard to safety data, adverse drug reactions occurred in $4.3 \%$ of the doripenem group and $4.0 \%$ of the meropenem group. These results demonstrated that both doripenem and meropenem have a high safety profile.

\section{Nosocomial pneumonia}

Several clinical studies have been conducted to examine the efficacy and safety of doripenem versus other antibiotics on the market in treating NP.

The first study was a Phase 3, multicenter, prospective, randomized, open-label study conducted by Réa-Neto et $\mathrm{al}^{33}$ to compare IV doripenem q8h 60-minute infusion versus intravenous piperacillin/tazobactam 4.5 g every 6 hours 30 minute infusion in non-ventilated NP patients and patients with early-onset ventilator-associated pneumonia (VAP). Four hundred forty-eight adult patients were enrolled, 225 were randomized to the doripenem group and 223 to the piperacillin/tazobactam group. Randomization was stratified by mechanical ventilation association, severity of illness, and 
geographic region. After 72 hours of IV study drug therapy, the patients were allowed to switch to oral levofloxacin $750 \mathrm{mg}$ daily if they met all the criteria, which indicated sufficient clinical improvement.

The primary endpoint was to compare the clinical cure rate of IV doripenem versus IV piperacillin/tazobactam at the TOC visit in patients with NP. The TOC visit was conducted 7 to 14 days after completion of study drug therapy. Secondary endpoint was to compare the clinical response rate and microbiological response rate of IV doripenem versus IV piperacillin/tazobactam and to compare the safety profile of the two antibiotics.

Clinical cure rates for meropenem and piperacillin/tazobactam were $67.6 \%$ and $67.4 \%$, respectively. Microbiological cure rate was $84.5 \%$ for doripenem versus $80.7 \%$ for piperacillin/tazobactam. The primary endpoint analysis revealed that doripenem was non-inferior to piperacillin/tazobactam in efficacy in treatment of NP. Secondary endpoint analyses demonstrated that doripenem was clinically and therapeutically noninferior to piperacillin/tazobactam.

The most common adverse drug reactions in the doripenem group were increased liver enzymes, thrombocythemia and diarrhea. Adverse events were comparable between doripenem (16.1\%) and piperacillin/tazobactam (17.6\%), and the authors concluded that both doripenem and piperacillin/ tazobactam have high safety profiles.

The second study was a Phase 3 prospective, multicenter, randomized open-label trial conducted by Chastre et $\mathrm{al}^{34}$ to compare IV doripenem $500 \mathrm{mg}$ every 8 hours 4-hour infusion versus IV imipenem $500 \mathrm{mg}$ every 6 hours 30 -minute infusion, or $1000 \mathrm{mg}$ every 8 hours 60 -minute infusion in treating adult patients with VAP. Duration of treatment was 7 to 14 days for both antibiotics. Number of patients enrolled was 531, 264 in the doripenem group and 267 in the imipenem group. Randomization was stratified by duration of mechanical ventilation, severity of illness and by region.

The study design for patients in the doripenem group to receive 4-hour infusion could be based on the Psathas et al study, ${ }^{35}$ which was able to demonstrate that, in vitro, doripenem was stable for up to 12 hours in $0.9 \% \mathrm{NaCl}$ solution at room temperature.

The primary endpoint was to compare the clinical response rate of IV doripenem versus IV imipenem at the TOC. The TOC was conducted 7 to 14 days after completion of study drug therapy. The secondary endpoint was to compare per subject microbiological response rate, per pathogen microbiological outcome rate, per pathogen clinical cure rate, and the safety profile of the two antibiotics.
Clinical cure rates in the ME group for doripenem and imipenem were $69.0 \%$ and $64.5 \%$, respectively. Favorable per-subject microbiological response rate in the ME at TOC visit for doripenem and imipenem was $73.3 \%$ and $67.3 \%$, respectively. The results demonstrated that doripenem was not inferior to imipenem in efficacy in treating VAP.

The most common drug related adverse reactions with doripenem were increased liver enzymes (4.6\%), diarrhea (1.9\%), rash (1.9\%), and vomiting (1.5\%). The incidence of adverse events was $17.2 \%$ for doripenem versus $17.5 \%$ for imipenem. Thus, doripenem was generally well tolerated and the safety profiles of doripenem and imipenem were comparable.

A Japanese study examined the clinical efficacy and safety of IV doripenem $250 \mathrm{mg}$ every 12 hours versus IV meropenem $500 \mathrm{mg}$ every 12 hours in a randomized, double-blind trial in treatment of patients with respiratory infections. ${ }^{36}$ The majority of the patients in both groups were patients with bacterial pneumonia (131/193), and the rest were patients with concomitant chronic respiratory tract and other lung diseases (eg, chronic bronchitis, bronchietasis, bronchial asthma or emphysema) (62/193). Both groups were treated for 7 days. One hundred and ninety-three patients were evaluated for clinical efficacy. Clinical efficacy was $92.7 \%$ and $90.7 \%$ in the doripenem group and the meropenem group, respectively. The authors concluded that doripenem demonstrated noninferiority to meropenem in clinical efficacy. For safety profile, 218 patients were available for data analysis. The incidence of nonlaboratory adverse drug reactions was $8.1 \%$ in the doripenem group and $6.5 \%$ in the meropenem group. For laboratory adverse drug reactions, the incidence was $23.4 \%$ and $25.5 \%$ in the doripenem group and meropenem group, respectively. The authors found no significant difference between nonlaboratory and laboratory adverse drug reaction incidence.

In summary, although doripenem has not yet been approved by the FDA for treating NP, the prospective clinical trials mentioned above found that doripenem was not inferior to other antibacterial agents on the market for treating cIAIs, cUTIs and NP in terms of efficacy and adverse effects.

Table 3 Manufacturer renal dosage adjustment recommendations ${ }^{10}$

\begin{tabular}{ll}
\hline Creatinine clearance & Dose \\
\hline$>50 \mathrm{~mL} / \mathrm{min}$ & No dosage adjustment \\
$\geq 30$ to $\leq 50 \mathrm{~mL} / \mathrm{min}$ & $\begin{array}{l}250 \mathrm{mg} \text { intravenously (over I hour) } \\
\text { every } 8 \text { hours }\end{array}$ \\
$>10$ to $<30 \mathrm{~mL} / \mathrm{min}$ & $250 \mathrm{mg}$ intravenously (over I hour) \\
& every I 2 hours \\
Dialysis & $\begin{array}{l}\text { Insufficient information to make } \\
\text { dosing recommendations }\end{array}$ \\
\hline
\end{tabular}




\section{Safety, drug interactions, and tolerability \\ Adverse reactions}

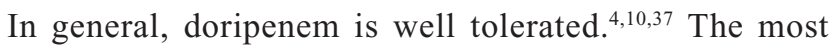
common adverse reactions reported in Phase 3 clinical trials included headache, nausea, diarrhea, rash, and phlebitis. ${ }^{20}$

Rare, but serious, doripenem postmarket adverse events reported included anaphylaxis, neutropenia, Stevens-Johnson Syndrome, toxic epidermal necrolysis, interstitial pneumonia, and seizure. ${ }^{10}$ Doripenem was noted to cause a mild elevation in alanine aminotransferase and aspartate aminotransferase enzymes. ${ }^{33}$ Doripenem has also caused pneumonitis when administered via inhalation, therefore, this route should be avoided. ${ }^{10}$

Previous carbapenems have been noted to induce seizures primarily via inhibition of $\gamma$-aminobutyric acid (GABA) receptor binding..$^{20,29,34,38}$ Doripenem has low affinity to the GABA receptor, resulting in low potential for seizure induction. ${ }^{34}$ In an experiment by Horiuchi et al utilizing rats and dogs, doripenem did not induce seizure-like behavior or activity on electroencephalogram (EEG). In addition, doripenem did not induce convulsive activities post intracerebroventricular injection. ${ }^{4,38}$ These findings are consistent with other Phase 3 clinical trials of doripenem. ${ }^{33}$

\section{Safety}

Doripenem should be used with caution or avoided in patients with previous hypersensitivity to $\beta$-lactam antibiotics. Severe anaphylaxis and serious skin reactions have developed in those patients with hypersensitivity to $\beta$-lactam antibiotics. ${ }^{4,10,39}$ Structural similarity of the bi-cyclic core of $\beta$-lactam antibiotics is thought to be the underlying cause of cross-reactivity. ${ }^{37,39}$ A retrospective chart review concluded that carbapenem hypersensitivity reaction occurred in $9 \%$ to $11 \%$ of patients with a history of penicillin allergy and $3 \%$ to $4 \%$ in patients without penicillin allergy. $4,37,39$

Due to lack of safety and efficacy data in the pediatric population, doripenem is only recommended for patients $\geq 18$ years of age. ${ }^{10,20}$ Imipenem has been approved for use in neonates; ertapenem and meropenem in infants $\geq 3$ months of age. ${ }^{20}$

In pregnancy, doripenem is classified as a category $\mathrm{B}$ medication as it did not produce teratogenic effects, ossification, or developmental delays. Animal reproduction studies have failed to demonstrate a fetal risk; however, there have been no controlled studies in pregnant women or animal studies demonstrating adverse effects. Doripenem should be used with caution in pregnant women and should be administered only if there are clear indications. ${ }^{10}$

\section{Drug interactions}

Doripenem should be used with caution when administered concomitantly with valproic acid due to the inhibition of valproic acid glucuronide hydrolysis leading to sub-therapeutic valproic serum levels resulting in possible seizure activity., ${ }^{4}, 10$ Valproic levels should be monitored carefully with concomitant doripenem use. ${ }^{10,20}$ A different antibiotic or anticonvulsant should be considered if the valproic level is not readily maintained in therapeutic range. ${ }^{10,20}$

Concomitant use of doripenem and probenecid should be avoided. ${ }^{10}$ Probenecid interferes with the active tubular secretion of doripenem resulting in an increased doripenem plasma concentration causing a $75 \%$ increase AUC and prolonged plasma elimination half life by $53 \% .^{10,20,33}$

\section{Place in therapy}

Generally, doripenem is well tolerated in patients with serious bacterial infections. The most common side effects include headache, nausea and diarrhea, while the most commonly reported laboratory abnormalities included increased levels of ALT and AST. ${ }^{14}$ There is less seizure potential as it has the lowest GABA receptor binding affinity among the carbapenems. ${ }^{34,38}$ Caution should be used in patients with known hypersensitivity to carbapenems or $\beta$-lactam agents.

Doripenem is used as a single agent for the treatment of cIAIs or complicated cUTIs in adult patients. ${ }^{18}$ Doripenem's dosing schedule makes it fairly easy to administer in hospitals, nursing homes, or other skilled nursing facilities. In limited circumstances, doripenem may even be used for qualified patients requiring outpatient (home) intravenous antibiotics.

Doripenem has demonstrated a decrease in the length of hospital stay and time of mechanical ventilation in a Phase 3, randomized, open-label, noninferiority study.$^{40}$ It combines the desirable attributes of imipenem and meropenem, which makes it a favorable drug against serious bacterial infections. It is well-tolerated and proven to be cost-effective leading to greater patient and hospital satisfaction. These factors make doripenem a broad-spectrum antibiotic that can be used to treat a wide range of infections while minimizing negative effects on patients.

Since carbapenems have time-dependent bactericidal effects, the optimal method to maximize the $\% \mathrm{~T}>\mathrm{MIC}$ is to give the drug as a continuous infusion. ${ }^{16,41}$ Alternative dosing strategies such as continuous or prolonged infusions of previous carbapenems (ie, imipenem and meropenem) have had limited feasibility due to their stability at room temperature for 4 to 6 hours. ${ }^{16}$ The prolonged stability of doripenem at room temperature may allow for prolonged infusions in an effort to extend the T > MIC and, therefore, enhance anti- 
Table 4 Adverse events reported with doripenem 500 mg IV every 8 hours for treatment in complicated urinary tract infections and intra-abdominal infections in three Phase 3 clinical trials ${ }^{20}$

\begin{tabular}{ll}
\hline Adverse event $(\mathbf{N}=\mathbf{8 5 3})$ & Frequency range (\%) \\
\hline Headache & $4-16$ \\
Nausea & $4-12$ \\
Diarrhea & $6-11$ \\
Anemia & $2-10$ \\
Phlebitis & $4-8$ \\
Rash & $0-5$ \\
Pruritus & $0-3$ \\
Hepatic enzyme elevation & $1-2$ \\
Vulvomycotic infection & $0-2$ \\
Oral candidiasis & $0-1$ \\
\hline
\end{tabular}

microbial activity. Ikawa et al reported that prolonging the infusion time (4 hours) was more effective in increasing the pharmacokinetic and pharmacodynamic breakpoint to achieve a T > MIC of $40 \% .{ }^{6}$ Van Wart et al used pharmacokineticpharmacodynamic modeling to show that a higher dose of doripenem $(1000 \mathrm{mg}$ ) infused over 4 hours every 8 hours was predicted to be effective for Gram-negative bacilli with MIC values of $8 \mathrm{mcg} / \mathrm{mL}$, which would currently be considered carbapenem nonsusceptible (MICs, $\geq 8 \mu \mathrm{g} / \mathrm{mL}$ ). ${ }^{42}$ Ongoing clinical trials are evaluating the use of this higher dose and prolonged infusion time for hospital-acquired pneumonia. ${ }^{42}$

\section{Conclusions}

Doripenem is a valuable drug that is being used more frequently in antibiotic therapy. It has proven its efficacy against NP, cIAIs and cUTIs. It is currently the latest FDA approved carbapenem and combines the antimicrobial activity of meropenem against Gram-negative bacteria and imipenem's action against Gram-positive organisms. Doripenem offers further solution to the treatment of nosocomial infections given its efficacy, spectrum, and $\beta$-lactamase stability compared to other carbapenems. ${ }^{2}$ Present data suggests that doripenem can play an important role in patients with serious nosocomial infections in the setting of multidrug resistant Gram-negative organisms where $P$. aeruginosa is prevalent. ${ }^{4}$

The efficacy and tolerability of doripenem in adults with cIAIs, cUTIs, and VAP, has been shown in several early trials. ${ }^{18,29,34}$

A recent study comparing resource utilization with doripenem versus imipenem from a hospital perspective among patients with VAP has demonstrated a significantly shorter length of stay and time on mechanical ventilation with doripenem. This study suggests that doripenem use may be economically and clinically beneficial to hospitals and patients. ${ }^{40}$ These desirable effects make doripenem a key drug to treat serious bacterial infections.

Doripenem's broad-spectrum of activity, its reported efficacy, and cost-effectiveness may make this the drug of choice for serious bacterial infections. Current clinical trials and in vitro susceptibilities have shown that doripenem may be useful for adult patients with serious infections that require broad spectrum antibiotics against multidrug resistant pathogens. ${ }^{43}$

In vitro testing demonstrated that doripenem exhibits low potential for selecting for resistance. ${ }^{14}$ One recent study examined 34 carbapenem-resistant $P$. aeruginosa isolates and found that doripenem displayed the lowest rates of resistance with an $\mathrm{MIC}_{50}$ value of $8 \mathrm{mg} / \mathrm{mL} .{ }^{19}$ Doripenem may soon be widely used in the hospital setting to treat serious polymicrobial infections.

However, doripenem's broad spectrum of activity and safety profile make it susceptible to misuse and overuse. ${ }^{44}$ This can result in higher resistance rates to common Gramnegative bacteria such as $P$. aeruginosa. Care should, therefore, be taken when considering doripenem as a first-line drug. Development of in vivo resistance against doripenem should be further investigated. ${ }^{4}$ Local hospital epidemiology and bacterial resistance patterns should be key factors in making clinical use decisions.

\section{Disclosures}

The authors declare no conflicts of interest.

\section{References}

1. Vergidis PI, Falagas ME, Shimada J, Yamaguchi K, Shiba T. Multidrugresistant Gram-negative bacterial infections: the emerging threat and potential novel treatment options. Curr Opin Investig Drugs. 2008;9(2):176-183.

2. Fritsche TR, Stilwell MG, Jones RN. Antimicrobial activity of doripenem (S-4661): a global surveillance report (2003). Clin Microbiol Infect. 2005;11(12):974-984.

3. Shimada J, Yamaguchi K, Shiba T, et al. [A new carbapenem antibiotic for injection: characteristics of doripenem]. Jpn J Antibiot. 2005;58(6):489-506.

4. Poulakou G, Giamarellou H. Doripenem: an expected arrival in the treatment of infections caused by multidrug-resistant Gram-negative pathogens. Expert Opin Investig Drugs. 2008;17(5):749-771.

5. Lo TS, Welch JM, Alonto AM, Vicaldo-Alonto EA. A review of the carbapenems in clinical use and clinical trials. Recent Pat Antiinfect Drug Discov. 2008;3(2):123-131.

6. Ikawa K, Morikawa N, Uehara S, et al. Pharmacokinetic-pharmacodynamic target attainment analysis of doripenem in infected patients. Int J Antimicrob Agents. 2009;33(3):276-279.

7. Weigelt JA. Empiric treatment options in the management of complicated intra-abdominal infections. Cleve Clin J Med. 2007; 74(Suppl 4):S29-S37.

8. Fukasawa M, Sumita Y, Harabe ET, et al. Stability of meropenem and effect of 1 beta-methyl substitution on its stability in the presence of renal dehydropeptidase I. Antimicrob Agents Chemother. 1992;36(7):1577-1579. 
9. Zhanel GG, Wiebe R, Dilay L, et al. Comparative review of the carbapenems. Drugs. 2007;67(7):1027-1052.

10. Package insert. Doribax ${ }^{\circledR}$ (doripenem for injection). Raritan, NJ: OrthoMcNeil Pharmaceuticals, Inc; 2008 Available from www.doribax. com/doribax/shared/pi/doribax.pdf Accessed: January 1, 2009.

11. Hagerman JK, Knechtel SA, Klepser ME. Doripenem: A new extendedspectrum carbapenem antibiotic. Formulary. 2007;42:676-688.

12. Nicolau DP. Carbapenems: a potent class of antibiotics. Expert Opin Pharmacother. 2008;9(1):23-37.

13. Greer ND. Doripenem (Doribax): the newest addition to the carbapenems. Proc (Bayl Univ Med Cent). 2008;21(3):337-341.

14. Bulletin. New Product, Doribax ${ }^{\circledR}$ (Doripenem for Injection). Washington, DC: American Pharmacists Association; 2008.

15. Matthews SJ, Lancaster JW. Doripenem monohydrate, a broad-spectrum carbapenem antibiotic. Clin Ther. 2009;31(1):42-63.

16. Kotapati S, Nicolau DP, Nightingale CH, Kuti JL. Clinical and economic benefits of a meropenem dosage strategy based on pharmacodynamic concepts. Am J Health Syst Pharm. 2004;61(12):1264-1270.

17. Katsube T, Yano Y, Yamano Y, et al. Pharmacokinetic-pharmacodynamic modeling and simulation for bactericidal effect in an in vitro dynamic model. J Pharm Sci. 2008;97(9):4108-4117.

18. Keam SJ. Doripenem: a review of its use in the treatment of bacterial infections Drugs. 2008;68(14):2021-2057.

19. Jones RN, Huynh HK, Biedenbach DJ. Activities of doripenem (S-4661) against drug-resistant clinical pathogens. Antimicrob Agents Chemother. 2004;48(8):3136-3140.

20. Cada D, Levien T, Mistry B, Baker D. Doripenem for Injection. Hospital Pharmacy. 2008;43(2):210-218

21. Nishio H, Komatsu M, Shibata N, et al. Metallo-beta-lactamase-producing gram-negative bacilli: laboratory-based surveillance in cooperation with 13 clinical laboratories in the Kinki region of Japan. J Clin Microbiol. 2004;42(11):5256-5263.

22. Jones RN, Sader HS, Fritsche TR. Comparative activity of doripenem and three other carbapenems tested against Gram-negative bacilli with various beta-lactamase resistance mechanisms. Diagn Microbiol Infect Dis. 2005;52(1):71-74.

23. Schwaber MJ, Carmeli Y. Carbapenem-resistant Enterobacteriaceae: a potential threat. JAMA. 2008;300(24):2911-2913.

24. Mushtaq S, Ge Y, Livermore DM. Comparative activities of doripenem versus isolates, mutants, and transconjugants of Enterobacteriaceae and Acinetobacter spp. with characterized beta-lactamases. Antimicrob Agents Chemother. 2004;48(4):1313-1319.

25. Li XZ, Nikaido H. Efflux-mediated drug resistance in bacteria. Drugs. 2004;64(2):159-204.

26. Mushtaq S, Ge Y, Livermore DM. Doripenem versus Pseudomonas aeruginosa in vitro: activity against characterized isolates, mutants, and transconjugants and resistance selection potential. Antimicrob Agents Chemother. 2004;48(8):3086-3092.

27. Jones RN, Sader HS, Fritsche TR, Janechek MJ. Selection of a surrogate beta-lactam testing agent for initial susceptibility testing of doripenem, a new carbapenem. Diagn Microbiol Infect Dis. 2007;59(4):467-472.

28. PRNewswire. "FDA requires additional information on Doribax for treatment of hospital-acquired pneumonia". Johnson \& Johnson Pharmaceuticals Research and Development, L.L.C.; August 21, 2008 Available at: www.drugs.com/nda/fda-requires-additional-doribaxhospital-acquired-pneumonia-1610.html Accessed: January 14, 2009.
29. Lucasti C, Jasovich A, Umeh O, et al. Efficacy and tolerability of IV doripenem versus meropenem in adults with complicated intraabdominal infection: a phase III, prospective, multicenter, randomized, double-blind, noninferiority study. Clin Ther. 2008;30(5):868-883.

30. Malafaia O, Umeh O, Jiang J. Doripenem versus meropenem for the treatment of complicated intra-abdominal infections [Abstract No. L-1564b plus poster]. 47th Interscience Conference on Antimicrobial Agents and Chemotherapy; 2006; San Francisco, CA, USA; 2006.

31. Naber K, Redman R, Kotey P, Llorens L, Kaniga K. Intravenous therapy with doripenem versus Levofloxacin with an option for oral step down therapy in the treatment of complicated urinary tract infections and pyelonephritis [Abstract No. 833 plus poster]. Proceedings of the 17th European Congress of Clinical Microbiology and Infectious Diseases and the 25th International Congress of Chemotherapy; 2007 March 31-April 3; Munich, Germany; 2007.

32. Kamidono S, Arakawa S, Hirose T. et al. Double-blind, controlled study to evaluate safety and efficacy of doripenem and meropenem in patients with complicated urinary tract infection [in Japanese]. Jpn J Chemother. 2005; 53(Suppl 1):244-259.

33. Rea-Neto A, Niederman M, Lobo SM, et al. Efficacy and safety of doripenem versus piperacillin/tazobactam in nosocomial pneumonia: a randomized, open-label, multicenter study. Curr Med Res Opin. 2008;24(7):2113-2126.

34. Chastre J, Wunderink R, Prokocimer P, et al. Efficacy and safety of intravenous infusion of doripenem versus imipenem in ventilatorassociated pneumonia: a multicenter, randomized study. Crit Care Med. 2008;36(4):1089-1096.

35. Psathas PA, Kuzmission A, Ikeda K, Yasuo S. Stability of doripenem in vitro in representative infusion solutions and infusion bags. Clin Ther. 2008;30(11):2075-2087.

36. Saito A, Watanabe A, Nakata K, et al. Comparative study of doripenem and meropenem in respiratory infections. Phase III double-blind comparative study [in Japanese]. Jpn J Chemother. 2005;53(Suppl. 1):185-204.

37. Lister PD. Carbapenems in the USA: focus on doripenem. Expert Rev Anti Infect Ther. 2007;5(5):793-809.

38. Horiuchi M, Kimura M, Tokumura M, et al. Absence of convulsive liability of doripenem, a new carbapenem antibiotic, in comparison with beta-lactam antibiotics. Toxicology. 2006;222(1-2):114-124.

39. Prescott WA Jr, Kusmierski KA. Clinical importance of carbapenem hypersensitivity in patients with self-reported and documented penicillin allergy. Pharmacotherapy. 2007;27(1):137-142.

40. Merchant S, Gast C, Nathwani D, et al. Hospital resource utilization with doripenem versus imipenem in the treatment of ventilator-associated pneumonia. Clin Ther. 2008;30(4):717-733.

41. Kuti JL, Maglio D, Nightingale CH, Nicolau DP. Economic benefit of a meropenem dosage strategy based on pharmacodynamic concepts. Am J Health Syst Pharm. 2003;60(6):565-568.

42. Van Wart SA, Andes DR, Ambrose PG, Bhavnani SM. Pharmacokineticpharmacodynamic modeling to support doripenem dose regimen optimization for critically ill patients. Diagn Microbiol Infect Dis. 2009;63(4):409-414.

43. Anderson DL. Doripenem. Drugs Today (Barc). 2006;42(6):399-404.

44. Volles DF, Branan TN. Antibiotics in the intensive care unit: focus on agents for resistant pathogens. Emerg Med Clin North Am. 2008;26(3):813-834.

Infection and Drug Resistance

\section{Publish your work in this journal}

Infection and Drug Resistance is an international, peer-reviewed openaccess journal that focuses on the optimal treatment of infection (bacterial, fungal and viral) and the development and institution of preventive strategies to minimize the development and spread of resistance. The journal is specifically concerned with the epidemiology of antibiotic

resistance and the mechanisms of resistance development and diffusion in both hospitals and the community. The manuscript management system is completely online and includes a very quick and fair peer-review system, which is all easy to use. Visit http://www.dovepress.com/ testimonials.php to read real quotes from published authors. 